PROCEEDINGS OF THE

AMERICAN MATHEMATICAL SOCIETY

Volume 135, Number 2, February 2007, Pages 507-515

S 0002-9939(06)08755-7

Article electronically published on August 28, 2006

\title{
RIGIDITY OF SURFACES WHOSE GEODESIC FLOWS PRESERVE SMOOTH FOLIATIONS OF CODIMENSION 1
}

\author{
JOSÉ BARBOSA GOMES AND RAFAEL O. RUGGIERO
}

(Communicated by Michael Handel)

\begin{abstract}
Let $S$ be a closed orientable surface. Assume that there exists a codimension one foliation $\mathcal{F}$ of class $C^{3}$ in the unit tangent bundle of $S$, whose leaves are invariant under the geodesic flow of $S$. Then, the curvature of $S$ is a nonpositive constant.
\end{abstract}

\section{INTRODUCTION}

The study of the relationships between the smoothness of foliations which are invariant under the geodesic flow and geometric rigidity has produced a beautiful body of work involving dynamical systems, foliation theory, Riemannian geometry and group actions. The seminal work of Hurder and Katok 25] showed how the Godbillon-Vey theory could be used to get rigidity results for the geometry of surfaces of negative curvature by assuming smoothness of the central foliations of the geodesic flow. The study of the rigidity associated to the smoothness of central foliations of Anosov flows defined in circle bundles was concluded by Ghys 14, who proves that compact surfaces whose geodesic flows are Anosov and have $C^{2}$ central foliations have constant negative curvature and classifies up to smooth conjugacies all Anosov flows in circle bundles whose central foliations are $C^{2}$. In higher dimensions, there are also rigidity results for compact manifolds of negative curvature under smoothness assumptions of the invariant foliations of the geodesic flow (Kanai 26, Katok-Feres [11, 12] for pinched negative curvature, Hamenstädt 21], Benoist-Foulon-Labourie [2]), but a complete classification of manifolds admitting Anosov geodesic flows whose central foliations are sufficiently smooth is still an open problem.

The present paper deals with surfaces whose geodesic flows preserve highly smooth codimension one foliations. The existence of such foliations is a very exceptional feature of the dynamics, and we might well expect to get some rigidity of the geometry as in the case of surfaces with Anosov geodesic flows. The analytic foliation by invariant tori of the geodesic flow of a flat metric in the torus is a particular case of Liouville's theorem about completely integrable Hamiltonian systems. The regularity of the central foliations of the geodesic flow in a surface of

Received by the editors September 14, 2005.

2000 Mathematics Subject Classification. Primary 53C24; Secondary 53C22, 57R30, 37D40.

Key words and phrases. Godbillon-Vey number, geodesic flow, rigidity, Anosov flow.

The first author was supported in part by CAPES of the Brazilian Government.

The second author was supported in part by CNPq of the Brazilian Government.

(C)2006 American Mathematical Society Reverts to public domain 28 years from publication 
constant negative curvature is a consequence of the smoothness of the action of the affine group in the unit tangent bundle of the surface, which is a quotient of the universal covering of $\operatorname{PSL}(2, \mathbb{R})$. The main result of this paper is the following.

Theorem A. Let $(S, g)$ be a compact, orientable $C^{\infty}$ surface whose geodesic flow $\varphi_{t}$ preserves a $C^{3}$ codimension one foliation $\mathcal{F}$, i.e., $\varphi_{t}(\mathcal{F}(\theta))=\mathcal{F}\left(\varphi_{t}(\theta)\right)$ for every $\theta$ in the unit tangent bundle of $(S, g)$, where $\mathcal{F}(\theta)$ is the leaf containing $\theta$. Then the curvature of $(S, g)$ is a nonpositive constant.

Let us give a sketch of the proof of Theorem A. We show first of all that the assumption of existence of an invariant codimension one foliation implies that the surface has no conjugate points. This is a consequence of a result due to Mañé [28] about continuous, Lagrangian invariant subbundles of the geodesic flow. Hence the surface is either a flat torus by Hopf [23], or the genus of the surface is greater than one. In this latter case, it is not hard to show that the invariant foliation has no compact leaves, and hence a result due to Ghys [16] implies that if the foliation is of class $C^{3}$, then it is $C^{3}$ conjugate with the central foliation of the geodesic flow of a metric of constant negative curvature in the surface. Now, the link between the smoothness of the foliation and the rigidity of the surface can be made through the Godbillon-Vey theory and the work of Mitsumatsu 29], who calculates the Godbillon-Vey number of a $C^{2}$ central foliation of an Anosov geodesic flow which is $C^{2}$ conjugate with the central foliation of the geodesic flow of a surface with constant negative curvature. Since Theorem A has no assumption on the dynamics of the geodesic flow, we cannot apply directly Mitsumatsu's result to conclude that the curvature of the surface is constant. So the last step of the proof of Theorem A is to generalize Mitsumatsu's calculation of the Godbillon-Vey number for the invariant foliation of Theorem A and to show that the curvature of the surface is constant if and only if Mitsumatsu's defect (see Section 3 for the definition) of the invariant foliation is zero.

Theorem A generalizes from the dynamics point of view the rigidity theorem of Ghys concerning Anosov geodesic flows with smooth central foliations. However, there is a subtle difference between the regularity of the central foliations required by the theorem of Ghys and the regularity of the invariant foliation in Theorem A: in the former case the regularity is $C^{2}$ and in the latter case the regularity is $C^{3}$. In fact, the work of Ghys [16] implies that we can replace the $C^{3}$ assumption on the invariant foliation by $C^{2}$ with Lipschitz $C^{2}$ derivatives (see Section 4 for details). Of course, Theorem A is false if we just assume that the foliation is $C^{1}$ because the central foliations of any Anosov geodesic flow in a compact surface are $C^{1}$ by the work of Hopf 24]. We do not know if Theorem A holds assuming that the foliation is $C^{2}$. However, we believe that the theorem should be true in this case as well.

\section{Preliminaries}

We shall denote by $(M, g)$ a pair formed by a $C^{\infty}$ manifold $M$ endowed with a $C^{\infty}$ Riemannian metric $g$. The universal covering of $M$ will be denoted by $\tilde{M}$, the covering map is $\Pi: \tilde{M} \longrightarrow M$, and the universal covering endowed with the pullback of the metric $g$ by the covering map $\Pi$ is $(\tilde{M}, \tilde{g})$. The tangent space at $p \in M$ is $T_{p} M$, and the unit tangent bundle of $(M, g)$ will be denoted by $T_{1} M$. The canonical coordinates in the unit tangent bundle are $(p, v)$, where $p \in M$ and 
$v \in T_{p} M$ is a unit tangent vector. The canonical projection $\pi: T M \longrightarrow M$ is the map defined by $\pi(p, v)=p$.

The geodesic flow of $(M, g)$ will be denoted by $\varphi_{t}: T_{1} M \longrightarrow T_{1} M$, and the geodesic of $(M, g)$ with initial point $p$ and initial tangent vector $v$ will be denoted by $\gamma_{(p, v)}(t)$. We shall assume that all geodesics are parametrized by arc length. The geodesic $\gamma_{(p, v)}(t)$ has no conjugate points if each Jacobi field along the geodesic has at most one zero. The Riemannian manifold $(M, g)$ has no conjugate points if $\gamma_{(p, v)}(t)$ has no conjugate points for every $(p, v) \in T_{1} M$.

There are many equivalent definitions of manifolds without conjugate points. In fact, $(M, g)$ has no conjugate points if and only if the exponential map at every point is nonsingular. Moreover, $(M, g)$ has no conjugate points if and only if every geodesic of $(\tilde{M}, \tilde{g})$ is globally minimizing. In other words, given a geodesic $\gamma \subset \tilde{M}$ parametrized by arc length, the curve $\gamma[a, b]$ is the unique geodesic joining $\gamma(a)$ and $\gamma(b)$, and hence, $\gamma[a, b]$ is the unique minimizer of the length of curves joining its endpoints for every $a<b$. Manifolds with nonpositive curvature are typical examples of manifolds without conjugate points, although there are examples of such manifolds where the curvature changes sign (see, for instance, 20, 6]). Compact manifolds whose geodesic flows are Anosov have no conjugate points, according to a famous theorem due to Klingenberg [27.

The global geometry of surfaces without conjugate points has many features in common with the global geometry of manifolds with nonpositive curvature. The well-known work of Morse [30] implies that every geodesic in the universal covering of a compact surface without conjugate points is shadowed by a geodesic of the hyperbolic plane. Geodesic rays in the universal covering of a compact surface without conjugate points diverge according to the work of Green [18, and the only metrics in the torus without conjugate points are the flat metrics (Hopf [23] proves this fact for dimension two, and Burago and Ivanov [3] show the same result in any dimension). Eberlein generalizes most of the global geometry features of surfaces without conjugate points of higher genus to visibility manifolds (for the definition we refer to [7, [1]) of any dimension. The later introduction by Thurston and Gromov 19 of the so-called hyperbolic groups shows that the same picture of the global geometry of surfaces without conjugate points and higher genus occurs in a much more general setting.

\section{LAGRANGIAN SUBBUNDLES AND THE RiCCATI EQUATION}

In this section we make the first step towards the proof of the main theorem: we show the relationship between the existence of codimension one, invariant foliations in $T_{1} M$ and manifolds without conjugate points. We recall briefly some basic notions concerning the so-called Riccati equation associated to Jacobi fields and Lagrangian subbundles of the geodesic flow (see, for instance, [28], 31]). Let $\langle\langle\rangle$, be the Sasaki metric in the unit tangent bundle of $(M, g)$. Given $\theta \in T_{1} M$, let us denote by $\mathcal{V}_{\theta} \subset T_{\theta} T_{1} M$ the vertical subspace of $T_{\theta} T_{1} M$ (namely, the kernel of $d \pi$, the differential of the canonical projection). Let us denote by $\mathbf{H}_{\theta} \subset T_{\theta} T_{1} M$ the horizontal subspace of $T_{\theta} T_{1} M$, and let $X_{\theta} \in T_{\theta} T_{1} M$ be the unit vector tangent to the direction of the geodesic flow, i.e., $\frac{d}{d t} \varphi_{t}(\theta)=X_{\varphi_{t}(\theta)}$ for every $t \in \mathbb{R}$. Recall that $X_{\theta} \in \mathbf{H}_{\theta}$ for every $\theta \in T_{1} M$, and that the vertical and the horizontal subspaces at $\theta$ 
are perpendicular with respect to the Sasaki metric. Let us consider the subspaces

$$
\begin{gathered}
N_{\theta}=\left\{v \in T_{\theta}\left(T_{1} M\right) ;\left\langle\left\langle v, X_{\theta}\right\rangle\right\rangle=0\right\}, \\
\mathcal{H}_{\theta}=\mathbf{H}_{\theta} \cap N_{\theta} .
\end{gathered}
$$

The differential of the geodesic flow preserves the bundle of subspaces $N_{\theta}$, and the differential $\Omega$ of the canonical one-form of the geodesic flow defines a symplectic two-form when restricted to each $N_{\theta}$, that is also invariant under the geodesic flow.

Definition 2.1. Let $(M, g)$ be a complete, smooth Riemannian manifold of dimension $n$. A continuous subbundle of subspaces $\theta \rightarrow L_{\theta}$, where $L_{\theta} \subset N_{\theta}$, is called Lagrangian if $\Omega(v, w)=0$ for every $v, w \in L_{\theta}$, and $\operatorname{dim}\left(L_{\theta}\right)=n-1$, for every $\theta \in T_{1} M$.

If the dimension of $M$ is two, every continuous bundle $L_{\theta} \subset N_{\theta}$ of dimension-one subspaces is Lagrangian. A Lagrangian bundle that is invariant under the action of the differential of the geodesic flow will be called an invariant Lagrangian bundle. The following result is due to R. Mañé (28]).

Theorem 2.2. Let $(M, g)$ be a compact Riemannian manifold. If there exists a continuous invariant Lagrangian bundle $\mathcal{E}$ in the tangent space of $T_{1} M$, then $(M, g)$ has no conjugate points.

In fact, the proof of Mañé's theorem consists in showing that

$$
\mathcal{E}_{\theta} \cap \mathcal{V}_{\theta}=\{0\}
$$

for every $\theta \in T_{1} M$. From this fact it is easy to show the following well-known result (see [31] for instance) that we state without proof.

Lemma 2.3. Let $(S, g)$ be a compact surface without conjugate points. Given a continuous Lagrangian subbundle $\mathcal{E}$ of $T T_{1} S$ that is invariant under the geodesic flow, there exists a continuous function $U: T_{1} S \longrightarrow \mathbb{R}$ such that

(1) the subspace $\mathcal{E}_{\theta}$ is the graph of the linear map $\bar{U}_{\theta}: \mathcal{H}_{\theta} \longrightarrow \mathcal{V}_{\theta}$ given by $\bar{U}_{\theta}(Z)=U(\theta) Z$ for every $\theta \in T_{1} S$;

(2) the function $u_{\theta}(t)$ given by $u_{\theta}(t)=U\left(\phi_{t}(\theta)\right)$ is a solution of the Riccati equation

$$
u^{\prime}+u^{2}+K=0
$$

for every $\theta \in T_{1} S$.

\section{Godbillon-Vey class and Mitsumatsu's Defect}

Let $(S, g)$ be a $C^{\infty}$, closed oriented surface of genus greater than 1 with Gaussian curvature function $K=K_{g}: S \rightarrow \mathbb{R}$. Let $\left\{\varphi_{t}\right\}$ be the geodesic flow and $X=X_{g}$

be the geodesic vector field. Let $W=\frac{\partial}{\partial \zeta}$ be a smooth vector field tangent to the vertical fibers of $T_{1} S$ whose norm is equal to one in the Sasaki metric. The goal of this section is to prove the following result.

Theorem 3.1. Let $\mathcal{F}$ be a $C^{2}$ foliation in $T_{1} S$ of codimension 1 whose leaves are invariant under the geodesic flow. Let $U: T_{1} S \longrightarrow \mathbb{R}$ be the Riccati operator 
associated to the foliation $\mathcal{F}$ defined in Lemma 2.1. Then,

(1) the Godbillon-Vey number of $\mathcal{F}$ can be written as

$$
g v(\mathcal{F})=4 \pi^{2} \chi(S)-3 \int_{T_{1} S}(W U)^{2} \omega \wedge \theta_{1} \wedge \theta_{2},
$$

where $\chi(S)$ is the Euler characteristic of $S$;

(2) the function $U$ is constant along the fibers, i.e., $W U \equiv 0$, if and only if the metric $g$ in $S$ has constant curvature $\leq 0$.

We shall define the number $3 \int_{T_{1} S}(W U)^{2} \omega \wedge \theta_{1} \wedge \theta_{2}$ to be Mitsumatsu's defect. This notation is based on the fact that the number $4 \pi^{2} \chi(S)$ is the Godbillon-Vey number of the central foliations of the geodesic flow in any compact, orientable surface of constant negative curvature, according to Roussarie. So Theorem 3.1 implies that the Godbillon-Vey number of a $C^{2}$, codimension one, invariant foliation is maximal if and only if the surface has constant negative curvature.

Following Mitsumatsu (29]), we describe briefly how to calculate the GodbillonVey number of a $C^{2}$ invariant, codimension one foliation $\mathcal{F}$ in $T_{1} S$ in terms of the solution $U$ of the Riccati equation defined in the previous section. Observe that the tangent bundle of the foliation $\mathcal{F}$ gives us an invariant Lagrangian subbundle, namely, $\mathcal{E}_{\theta}=T_{\theta} \mathcal{F}_{\theta} \cap N_{\theta}$. So we shall consider throughout the section the operator $U$ described in Lemma 2.3 associated to the subbundle $\mathcal{E}$.

To begin with the calculation, let us recall Cartan's formalism for two-dimensional Riemannian geometry. Let $\omega$ be the connection 1-form in $T_{1} S$, and let $\theta_{1}$ and $\theta_{2}$ be the canonical 1-forms satisfying the Cartan structural equations (see, for example, 33]

$$
\begin{gathered}
\left\{\begin{array}{c}
d \theta_{1}=\omega \wedge \theta_{2}, \\
d \theta_{2}=-\omega \wedge \theta_{1},
\end{array}\right. \\
d \omega=-(K \circ \pi) \theta_{1} \wedge \theta_{2} .
\end{gathered}
$$

Let $\{W, X, Y\}$ be the orthonormal dual basis, where $W$ (dual to $\omega$ ) is the vertical vector field, and $X$ is the geodesic vector field. Then, $X$ and $Y$ span the horizontal space.

The structural equations in dual form are

$$
\begin{gathered}
{[Y, W]=X, \quad[W, X]=Y,} \\
{[X, Y]=K W .}
\end{gathered}
$$

Since $\mathcal{E}_{\theta}$ is the graph of the map $U_{\theta}: \mathcal{H}_{\theta} \rightarrow \mathcal{V}_{\theta}$, and the subbundle $\mathcal{E}$ is invariant under the geodesic flow, the function $u_{\theta}(t)=U\left(\phi_{t}(\theta)\right)$ is the slope of the subspace $d_{\theta} \varphi_{t}\left(E_{\theta}\right)$ in the plane $N_{\phi_{t}(\theta)}$ for every $\theta \in T_{1} S$. The Riccati equation, viewed as a differential equation in $T_{1} S$, can be written as

$$
X U+U^{2}+K \circ \pi=0 .
$$

Therefore, the tangent space $T_{\theta} \mathcal{F}_{\theta}$ of the leaf $\mathcal{F}_{\theta}$ at the point $\theta$ is spanned by $X_{\theta}$ and $Y_{\theta}+U(\theta) W_{\theta}$, and the 1-form

$$
\beta=\omega-U \theta_{2}
$$

defines $\mathcal{F}$. 
We have that

$$
\begin{aligned}
d \beta & =d \omega-(d U) \wedge \theta_{2}-U \cdot d \theta_{2}=-\left(k \circ \pi_{S}\right) \theta_{1} \wedge \theta_{2}-(d U) \wedge \theta_{2}-U\left(-\omega \wedge \theta_{1}\right) \\
& =-\left(k \circ \pi_{S}\right) \theta_{1} \wedge \theta_{2}-(d U) \wedge \theta_{2}+U \cdot \omega \wedge \theta_{1} .
\end{aligned}
$$

If we set $\eta=(W U) \cdot \theta_{2}-U \cdot \theta_{1}$, then

$$
\eta \wedge \beta=d \beta
$$

and

$$
\eta \wedge d \eta=\left[-2(W U)^{2}+U\left(W^{2} U\right)-U^{2}\right] \omega \wedge \theta_{1} \wedge \theta_{2} .
$$

As in [29], we get, using the Riccati equation and the Gauss-Bonnet theorem,

$$
g v(\mathcal{F})=4 \pi^{2} \chi(S)-3 \int_{T_{1} S}(W U)^{2} \omega \wedge \theta_{1} \wedge \theta_{2}
$$

where $\chi(S)$ is the Euler characteristic of $S$, thus proving item (1) of Theorem 3.1.

To show item (2) of Theorem 3.1, let us first observe that constant, negative curvature implies that $W U=0$. This is due to the fact that the unique codimension 1 foliations which are invariant under the geodesic flow in this case are the stable and the unstable central foliations, whose Godbillon-Vey number is precisely $4 \pi^{2} \chi(S)$ by the work of Roussarie. Therefore, the integral term in the formula of item 1 of Theorem 3.1 has to be zero and since the integrand is continuous and nonnegative it has to vanish everywhere in the unit tangent bundle. So we have to show that if $W U=0$, then the curvature of $(S, g)$ is constant.

Claim. If $W U=0$, then the function $U$ is constant.

Let us sketch the proof of this assertion. We would like to point out that in 29. the proof of the Claim in the Anosov case used the existence of the stable and the unstable solutions of the Riccati equation. The new fact in our argument is that the existence of just one smooth solution of the Riccati equation already implies that such a solution must be constant. Since the function $U$ is constant in the vertical fibers, we have that $U(p, v)=U(p,-v)$ for every $(p, v) \in T_{1} S$. Since $\pi\left(\phi_{t}(p, v)\right)=\pi\left(\phi_{-t}(p,-v)\right)$ we have that $U\left(\phi_{t}(p, v)\right)=U\left(\phi_{-t}(p,-v)\right)$ for every $(p, v) \in T_{1} S$ and $t \in \mathbb{R}$. Let us denote $\theta=(p, v)$ and $-\theta=(p,-v)$. According to the above equations, the solutions $u_{\theta}(t), u_{-\theta}(t)$ of the Riccati equation along the orbits $\phi_{t}(\theta), \phi_{-t}(-\theta)$ respectively, satisfy

$$
u_{\theta}(t)=u_{-\theta}(-t),
$$

or equivalently,

$$
u_{\theta}(-t)=u_{-\theta}(t),
$$

for every $\theta \in T_{1} S$ and $t \in \mathbb{R}$. Taking derivatives with respect to $t$ in both sides of the equation we get $-\frac{d}{d t} u_{\theta}(-t)=\frac{d}{d t} u_{-\theta}(t)$, and since $u_{-\theta}(t)$ is a solution of the Riccati equation, we get

$$
-u_{\theta}^{\prime}(-t)+u_{\theta}^{2}(-t)+K(-t)=0,
$$

for every $t \in \mathbb{R}$. Analogously, $u_{\theta}$ is a solution of the Riccati equation, so we have

$$
u_{\theta}^{\prime}(-t)+u_{\theta}^{2}(-t)+K(-t)=0
$$

for every $t \in \mathbb{R}$. Subtracting the two last equations we obtain $u_{\theta}^{\prime}(-t)=0$ for every $t \in \mathbb{R}$, and therefore, $u_{\theta}(t)$ is constant with respect to $t$ for every $\theta \in T_{1} S$. In other words, the function $U$ is constant along the orbits of the geodesic flow in $T_{1} S$. 
Now, as Mitsumatsu observes in 29, any two points in the unit tangent bundle $T_{1} S$ can be joined by a continuous path formed by three smooth curves, each of which is either a subset of an orbit of the geodesic flow or a subset of some vertical fiber. This clearly yields that $U$ is constant in $T_{1} S$.

From the claim and the Riccati equation, we have that $K(p)=-U^{2}(p, v)$ for every $p \in S$, and any unit vector $v \in T_{p} S$, thus concluding the proof of the theorem.

\section{The Proof of Theorem A}

Let $(S, g)$ be a compact, orientable surface whose geodesic flow preserves a codimension one, $C^{3}$ foliation $\mathcal{F}$. By Theorem $2.1(S, g)$ has no conjugate points, so it is either a flat torus or a surface of genus greater than one. If $(S, g)$ is the flat torus, Theorem A holds, so we are left to consider surfaces of genus greater than one.

Lemma 4.1. Let $\mathcal{F}$ be a $C^{1}$ codimension 1 foliation of $T_{1} S$ which is invariant under the geodesic flow of $(S, g)$. Then $\mathcal{F}$ has no compact leaves.

Proof. The proof is very simple and we make a sketch for the sake of completeness. Let $F_{0}$ be a compact leaf of $\mathcal{F}$. Since $F_{0}$ is invariant under the geodesic flow it carries a nonsingular vector field and hence $F_{0}$ is a torus. Moreover, by Theorem 1.1, $F_{0}$ is transversal to the vertical fibers of $T_{1} S$ and therefore the restriction to $F_{0}$ of the canonical projection $\pi: T_{1} S \longrightarrow S$ is a covering map. This implies that the canonical projection $\pi\left(F_{0}\right)$ is a compact surface without boundary in $S$, so the restriction of $\pi$ to $F_{0}$ has to be surjective and hence a covering map. This is clearly impossible since a torus cannot be a covering of a surface of genus greater than 1.

Now, we can use the following result due to E. Ghys ([16, Theorem 5.3).

Theorem 4.2. Let $\mathcal{F}$ be a $C^{r}$ codimension 1 foliation without compact leaves of the unit tangent bundle of a compact surface $S$ of genus greater than 1 , where $r \geq 3$. Then $\mathcal{F}$ is $C^{r}$ conjugate with the central foliation of the geodesic flow of a metric with constant curvature -1 in $S$.

By this theorem and [25], the Godbillon-Vey number of $\mathcal{F}$ equals the GodbillonVey number of a hyperbolic central foliation, so we can apply item (2) of Theorem 3.1 to conclude that the curvature of $(S, g)$ is constant. This finishes the proof of Theorem A.

Remark. Ghys in [16] observes that Theorem 4.2 holds if $\mathcal{F}$ is $C^{2}$ and has Lipschitz $C^{2}$ derivatives. Since a $C^{2}$ conjugacy with a hyperbolic central foliation is enough to apply Gobdillon-Vey theory, Theorem A remains true if we assume that $\mathcal{F}$ satisfies this weaker hypothesis.

\section{REFERENCES}

1. Ballmann, W., Gromov, M., Schröeder, V., Manifolds of Non-positive curvature, Boston, Birkhäuser, 1985. MR0823981 (87h:53050)

2. Benoist, Y., Foulon, F., Labourie, F., Flots d'Anosov à distribution stable et instable différentiable, J. Amer. Math. Soc. 1 (1992), 33-74. MR1124979 (93b:58112)

3. Burago, D., Ivanov, S., Riemannian tori without conjugate points are flat, Geom. Funct. Anal. 4 (1994), n. 3, 259-269. MR 1274115 (95h:53049)

4. Camacho, C., Lins, A., Teoria Geométrica das Folheações, CNPq, Brasília, 1979. MR0642831 (84m:57017) 
5. Candel, A., Conlon, L., Foliations I, American Mathematical Society, Providence, 2000. MR 1732868 (2002f:57058)

6. Donnay, V., Pugh, C., Anosov geodesic flows for embedded surfaces, Astérisque 287 (2003), 61-70. Geometric methods in dynamics (II), Volume in honor of Jacob Palis. De Melo, Viana, Yoccoz, Editors. MR2040000 (2005b:37051)

7. Eberlein, P., Geodesic flow in certain manifolds without conjugate points, Transactions of the American Mathematical Society 167 (1972), 151-170. MR0295387 (45:4453)

8. Eberlein, P., When is a geodesic flow of Anosov type? I, J. Differential Geometry 8 (1973), 437-463. MR0380891 (52:1788)

9. Eberlein, P., Horocycle flows on certain surfaces without conjugate points, Transactions of the American Mathematical Society 233 (1977), 1-36. MR0516501 (58:24380)

10. Ehresmann, C., Les connexions infinitésimales dans un espace fibré différentiable, Colloque de Topologie, CBRM, Bruxelles, 1950, pp. 29-55. MR0042768(13:159e)

11. Feres, R., Katok, A., Invariant tensor fields of dynamical systems with pinched Lyapunov exponents and rigidity of geodesic flows, Ergodic Theory and Dynamical Systems 9 (1989), 427-432. MR1016661 (90k:58167)

12. Feres, A., Katok, A., Anosov flows with smooth foliations and rigidity of geodesic flows in three-dimensional manifolds of negative curvature, Ergodic Theory and Dynamical Systems 10 (1990), 657-670. MR1091420 (92e:58150)

13. Ghys, E., Flots d'Anosov sur les 3-variétés fibrées en cercles, Ergodic Theory and Dynamical Systems 4 (1984), 67-80. MR0758894 (86b:58098)

14. Ghys, E., Flots d'Anosov dont les feuilletages stables sont différentiables, Ann. Scient. École Norm. Sup. 20 (1987), 251-270. MR0911758 (89h:58153)

15. Ghys, E., Déformations de flots d'Anosov et de groupes Fuchsiens, Ann. Inst. Fourier 42 (1992), 209-247. MR 1162561 (93j:58111)

16. Ghys, E., Rigidité Différentiable des Groupes Fuchsiens, Publications Mathématiques I.H.E.S. 78 (1993), 163-185. MR1259430 (95d:57009)

17. Godbillon, C., Vey, J., Un invariant des feuilletages de codimension 1, C. R. Acad. Sci. Paris 273 (1971), 92-95. MR0283816 (44:1046)

18. Green, L. W., Surfaces without conjugate points, Transactions of the American Mathematical Society 76 (1954), 529-546. MR0063097 (16:70d)

19. Gromov, M., Hyperbolic Groups, In: S. M. Gersten (Ed.), Essays in Group Theory, MSRI Publ., 8, Springer-Verlag, New York, 1987, pp. 75-264. MR0919826 (88e:20004)

20. Gulliver, R., On the variety of manifolds without conjugate points, Trans. Amer. Math. Soc. 210 (1975), 185-201. MR0383294 (52:4175)

21. Hamenstädt, U., Invariant two-forms for geodesic flows, Math. Ann. 301 (1995), 677-698. MR.1326763 (96a:58145)

22. Hedlund, G. A., Fuchsian groups and transitive horocycles, Duke Mathematical Journal II (1936), 530-542. MR 1545946

23. Hopf, E., Closed surfaces without conjugate points, Proceedings of the National Academy of Sciences of the United States of America 34 (1948), 47-51. MR0023591 (9:378d)

24. Hopf, E., Statistik der geodätischen Linien in Mannigfaltigkeiten negativer Krümmung, Ber. Verh. Sächs. Akad. Wiss. Leipzig 91, (1939). 261-304. MR0001464 (1:243a)

25. Hurder, S., Katok, A., Differentiability, rigidity and Godbillon-Vey classes for Anosov flows, Publications Mathématiques I.H.E.S. 72 (1990), 5-61. MR1087392(92b:58179)

26. Kanai, M., Geodesic flows of negatively curved manifolds with smooth stable and unstable foliations, Ergodic Theory and Dynamical Systems 8 (1988), 215-239. MR0951270|(89k:58230)

27. Klingenberg, W., Riemannian Geometry, Walter de Gruyter \& Co., Berlin, 1982. MR0666697 (84j:53001)

28. Mañé, R., On a theorem of Klingenberg, In: M. I. Camacho, M. J. Pacífico, F. Takens (Ed.), Dynamical Systems and Bifurcation Theory, Longman Scientific \& Technical, New York, 1987, pp. 319-345. MR0907897 (88k:58129)

29. Mitsumatsu, Y., A relation between the topological invariance of the Godbillon-Vey invariant and the differentiability of Anosov foliations, Advanced Studies in Pure Mathematics 5 (1985), 159-167. MR0877331 (88a:57050)

30. Morse, H. M., A fundamental class of geodesics on any closed surface of genus greater than one, Trans. American Math. Society 26 (1924), 25-60. MR.1501263

31. Paternain, G. P., Geodesic Flows, Birkhäuser, Boston, 1999. MR1712465 (2000h:53108) 
32. Raby, G., Invariance des classes de Godbillon-Vey par $C^{1}$-diffeomorphismes, Ann. Inst. Fourier 38(1) (1998), 205-213. MR0949006 (89j:57023)

33. Singer, I. M, Thorpe, J. A., Lecture Notes on Elementary Topology and Geometry, SpringerVerlag, New York, 1967. MR0213982 (35:4834)

Departamento de Matemática, Universidade Federal de Juiz de Fora, Juiz de Fora, MG, BrazIL, 36036-330

E-mail address: jbarbosa@ice.ufjf.br

Departamento de Matemática, Pontifícia Universidade Católica do Rio de Janeiro, Rio de Janeiro, RJ, Brazil, 22453-900

E-mail address: rorr@mat.puc-rio.br 